\title{
Is the CZT technology the future of nuclear cardiology?
}

\author{
Milena J. Henzlova, ${ }^{a}$ and Lane Duvall ${ }^{\mathrm{b}}$ \\ a Mount Sinai Health System, New York, NY \\ b Hartford Hospital, Hartford, CT
}

Received Sep 4, 2020; accepted Sep 4, 2020

doi: 10.1007/s12350-020-02399-4

\section{See related article, pp. 727-736}

The history of Nuclear Cardiology is well known, well documented, and occurred in living memory. Many of its pioneers are either still active or available to recount the imaginative first steps, the era of competition-free adoption, introduction of Tc-99m-based tracers, and acceptance and refinement of the testing protocols. ${ }^{1}$ Despite of the emergence of new non-invasive imaging methods for the detection of epicardial coronary artery disease (CAD), namely PET, stress echocardiography, and coronary CTA, SPECT imaging still remains the most often performed test for its proven diagnostic and prognostic value. ${ }^{2}$

During the last decade of the last century, stress methods were expanded by the introduction of potent coronary vasodilators (dipyridamole, adenosine, and regadenoson). The original SPECT tracer Tl-201 was more or less replaced with Tc-99m-based agents. Amazingly, the original hardware technology did not change substantially since its inception more than 50 years ago: Anger cameras consisting of $\mathrm{NaI}$ crystal detectors connected to an array of photo-multiplying tubes converting photons to electrical potentials underwent only minor improvements over the decades. Poor photon detection efficiency, long imaging times, QC requiring substantial resources and expertise, as well as new concerns about potential cancer hazards in the era of sky rocketing volume of external radiation diagnostic procedures, all called for new approaches. ${ }^{3}$

\footnotetext{
Reprint requests: Lane Duvall, Hartford Hospital, Hartford, CT; Lane.Duvall@hhchealth.org

J Nucl Cardiol 2022;29:737-40.

$1071-3581 / \$ 34.00$

Copyright (c) 2020 American Society of Nuclear Cardiology.
}

The most successful innovation available in more than a decade was the development and availability of CZT (cadmium-zinc-telluride) solid-state SPECT cameras. There are currently two commercially available models: D SPECT (Spectrum Dynamics, Caesarea, Israel) and Discovery 530c (GE Healthcare). Simultaneous acquisition (no moving parts) of the photons originating mostly from the imaged object (the myocardium) thanks to focused collimation increases the camera efficiency 5-10 times. CZT cameras allow for fast acquisition (3-5 vs 20-30 minutes), substantial tracer dose reduction (from $>12$ to $5-7 \mathrm{mSv}$ per test), time savings, and simplified QC procedures. ${ }^{4}$

The performance of the two available CZT cameras is not identical. However, the advantages compared to the Anger camera are similar for both models: spatial resolution and contrast-to-noise ratio are better with the Discovery NM 530c; count sensitivity is higher with the D SPECT. ${ }^{5}$ Initial hesitancy to embrace the new technology has several explanations: (1) substantially higher initial purchase cost (2-3 times compared to the traditional camera), (2) lack of clinical data-no clinical comparative studies preceded the introduction of the new technology, (3) no track record of reliability, maintenance, and longevity of the CZT technology, and (4) cardiac-only imaging ability.

Now in 2020, enough evidence has been collected and published from multiple laboratories in many countries on several continents, to allow the designation of CZT technology as the "gold standard" of SPECT imaging. Methodology for dosing, imaging, and attenuation correction or mitigation has been established. ${ }^{6}$ CZT imaging allows for substantially lower internal radiation dose to the patients, subsequently to the laboratory personnel, as well as to the general public. ${ }^{7}$ The quality of CZT MPI images is in most cases superior to the traditional $\mathrm{NaI}$ images. ${ }^{8}$ Negative aspects include lack of motion correction (compensated by speed of imaging and ease of re-imaging) and limited options for 
attenuation correction or mitigation (by combination with CT or using multi-positional imaging). CZT technology now allows for determination of coronary flow reserve (CFR) and of absolute coronary flow, which was so far a domain of PET imaging. ${ }^{9,10}$

A qualitative and quantitative milestone in the developing CZT story is the design and execution of an observational study REFINE SPECT (REgistry of Fast Myocardial Perfusion Imaging with NExt generation SPECT). This endeavor will likely become a landmark in diverse aspects of MPI using CZT imaging technology. ${ }^{11}$ REFINE SPECT, a multicenter, international registry, collected clinical, imaging, and prognostic data from 20,418 patients from 5 institutions, using both available CZT models, both available Tc-99m tracers (sestamibi and tetrofosmin), all available stressors (48\% exercise and $52 \%$ pharma stress), and all practiced protocols (reststress, stress-rest, stress-only, and 2-day tests). A diagnostic arm of the study collected 2,079 studies from 9 centers. Image evaluations were done visually on site and additionally by automated quantitative perfusion analysis by the Core Laboratory (Cedars-Sinai Medical Center Los Angeles, CA), which developed and validated the process. Automated image reading is now within the reach of routine clinical application. ${ }^{12}$ First papers and abstracts from the Registry started appearing in 2019. Available so far is data of diagnostic and prognostic utility of CZT SPECT from the whole Registry ${ }^{13}$ and from the subgroup of diabetics. ${ }^{14}$ Limits of transient ischemic dilation (TID) for CZT detectors were established. ${ }^{15}$

The next paper in the REFINE series is published in the current issue of $J N C \circledast{ }^{16}$ The prognostic registry cohort was divided according to the patient's BMI into 3 groups (BMI $<30,30-35$, and $>35 \mathrm{~kg} / \mathrm{m}^{2}$ ). All patients were prospectively followed for the development of MACE, which included all-cause mortality, non-fatal MI, unstable angina (UA), or late revascularization defined as greater than 90 days after the CZT SPECT MPI. The mean follow-up time was 4.6 years. Visual interpretation (done on site) classified MPI results as normal, probably normal, equivocal, or abnormal or semi-quantitatively using the 17-segment model for determination of the SSS (summed stress score): normal $(\mathrm{SSS}=0)$, probably normal $(\mathrm{SSS}=1)$, equivocal $(\mathrm{SSS}=$ $2-3$ ), or abnormal (SSS $\geq 4$ ). The Core Laboratory, using automated image analysis, determined the TPD (total perfusion defect), which includes the extent and severity of all perfusion abnormalities). The patients were divided into 4 categories according to the extent of perfusion defects (TPD $<1 \%, 1 \%-5 \%, 5 \%-10 \%$, or $>$ $10 \%)$. The automated assessments used either single acquisition position (upright or supine) or two imaging positions (upright and supine for the D SPECT camera or supine and prone for the Discovery 530c).
The analysis found that the MACE incidence was related to the extent of the TPD, that quantitative reads had a stronger prognostic value compared to visual reads, and that two position acquisition was most useful in the highest BMI category $\left(>35 \mathrm{~kg} / \mathrm{m}^{2}\right)$. The results confirm the relationship between size and severity of the perfusion abnormalities and prognosis (MACE frequency) as convincingly as has been established using traditional NaI SPECT cameras.

The strength and novelty of the current study includes the following:

(1) Confirmation of the feasibility of abbreviated imaging time and significantly lower radiation doses using CZT SPECT cameras (average dose was 7.2 $\mathrm{mSv}$ ) in this unfortunately currently very common patient group-those with a BMI $>30 \mathrm{~kg} / \mathrm{m}^{2}$.

(2) Proof that limited access to CT attenuation correction can be mitigated by multi-position imaging (prone-supine or upright-supine), which requires relatively little time to perform.

(3) The value of automated reads was again demonstrated. In the foreseeable future this is likely to become the method of choice for unbiased reads of large data sets used for multiple purposes (such as clinical trials, novel tracers, comparative studies, or prognostication). The availability of automated image reads could in the future replace the need for the presence of a qualified reader to review stress images prior to triage to "stress-only imaging" or for in-person review of a completed test prior to the patient's discharge from the laboratory.

(4) Data were obtained prospectively from multiple laboratories, from diverse populations and included both types of available CZT cameras and employed all currently used Tc-99m protocols (rest-stress, stress-rest, stress-only, and 2-day tests). The generalization of the results is therefore more robust, than data collected in a single laboratory.

Newly introduced products (tracers, stressors, or new software) traditionally underwent some form of comparison with previously used products. This was not the case with CZT SPECT cameras. They became commercially available with assurances that the selfevident physics characteristics (markedly improved efficiency) do not need trial proof. Consequently, early adopters filled the void. Between 2010 and 2018, numerous papers (mostly from Europe, which has a proportionately higher number of CZT cameras) compared the diagnostic and prognostic strength of this new technology (Table 1). All of these studies with the exception of one meta-analysis are from single centers, most are retrospective, and the majority did not use 


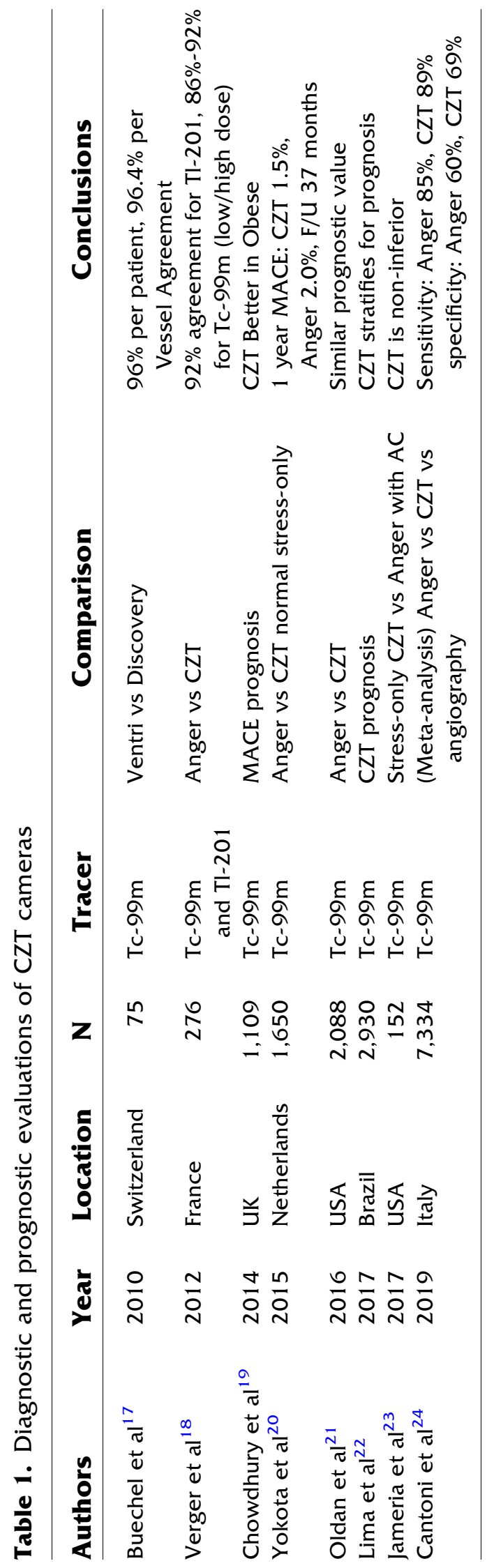

uniform imaging protocols and data collection. Despite those limitations, the conclusions are consistent: CZT technology is at least non-inferior, but likely superior, to the traditional (Anger) technology, while offering shorter imaging time and lower radiation dose.

The REFINE SPECT Registry contains an enormous amount of clinical, imaging, and prognostic data. It is with hopeful expectations that additional papers will be available in the near future. Perhaps the investigators should solicit requests for further data mining from the Nuclear Cardiology community as a whole.

\section{Disclosures}

The authors have nothing to disclose in relation to this manuscript.

\section{References}

1. Zaret BL. Nuclear cardiology: History and milestones. In: Iskandrian AE, Garcia EV, editors. Nuclear cardiac imaging: Principles and applications. 5th ed. Oxford: Oxford University Press; 2015. p. 3-10.

2. McNulty EJ, Hung YY, Almers LM, Go AS, Yeh RW. Population trends from 2000-2011 in nuclear myocardial perfusion imaging use. JAMA 2014;311:1248-9.

3. Einstein AJ, Henzlova MJ, Rajagopalan S. Estimating risk of cancer associated with radiation exposure from 64-slice computed tomography coronary angiography. JAMA 2007;298:317-23.

4. Esteves FP, Raggi P, Folks RD, Keidar Z, Askew JW, Rispler S, et al. Novel solid-state-detector dedicated cardiac camera for fast myocardial perfusion imaging: Multicenter comparison with standard dual detector cameras. J Nucl Cardiol 2009;16:927-34.

5. Imbert L, Poussier S, Franken PR, Songy B, Verger A, Morel O, et al. Compared performance of high-sensitivity cameras dedicated to myocardial perfusion SPECT: A comprehensive analysis of phantom and human images. J Nucl Med 2012;53:1897-903.

6. Henzlova MJ, Duvall WL, Einstein AJ, Travin MI, Verberne HJ. ASNC imaging guidelines for SPECT nuclear cardiology procedures: Stress, protocols, and tracers. J Nucl Cardiol 2016;23:60639.

7. Duvall WL, Guma KA, Kamen J, Croft LB, Parides M, George T, et al. Reduction in occupational and patient radiation exposure from myocardial perfusion imaging: Impact of stress-only imaging and high-efficiency SPECT camera technology. J Nucl Med 2013;54:1251-7.

8. Agostini D, Marie PY, Ben-Haim S, Rouzet F, Songy B, Giordano A, et al. Performance of cardiac cadmium-zinc-telluride gamma camera imaging in coronary artery disease: A review from the Cardiovascular Committee of the European Association of Nuclear Medicine (EANM). Eur J Nucl Med Mol Imaging 2016;43:2423-32.

9. Nkoulou R, Fuchs TA, Pazhenkottil AP, Kuest SM, Ghadri JR, Stehli J, et al. Absolute myocardial blood flow and flow reserve assessed by gated SPECT with cadmium-zinc-telluride detectors using ${ }^{99 \mathrm{~m}} \mathrm{Tc}$-tetrofosmin: Head-to-head comparison with ${ }^{13} \mathrm{~N}$-ammonia PET. J Nucl Med 2016;57:1887-92.

10. Agostini D, Roule V, Nganoa C, Roth N, Baavour R, Parienti JJ, et al. First validation of myocardial flow reserve assessed by 
dynamic ${ }^{(99 \mathrm{~m})}$ Tc-sestamibi CZT-SPECT camera: Head to head comparison with ${ }^{(15)} \mathrm{O}$-water PET and fractional flow reserve in patients with suspected coronary artery disease. The WATERDAY study. Eur J Nucl Med Mol Imaging 2018;45:1079-90.

11. Slomka PJ, Betancur J, Liang JX, Otaki Y, Hu LH, Sharir T, et al. Rationale and design of the REgistry of Fast Myocardial Perfusion Imaging with NExt generation SPECT (REFINE SPECT). J Nucl Cardiol 2020;27:1010-21.

12. Hu LH, Betancur J, Sharir T, Einstein AJ, Bokhari S, Fish MB, et al. Machine learning predicts per-vessel early coronary revascularization after fast myocardial perfusion SPECT: Results from multicentre REFINE SPECT Registry. Eur Heart J Cardiovasc Imaging 2020;21:549-59.

13. Otaki Y, Betancur J, Sharir T, Hu LH, Gransar H, Liang JX, et al. 5-Year prognostic value of quantitative versus visual MPI in subtle perfusion defects: Results from REFINE SPECT. JACC Cardiovasc Imaging 2020;13:774-85.

14. Han D, Rozanski A, Gransar H, Sharir T, Einstein AJ, Fish MB, et al. Myocardial ischemic burden and differences in prognosis among patients with and without diabetes: Results from the Multicenter International REFINE SPECT Registry. Diabetes Care 2020;43:453-9.

15. Miller RJH, Hu LH, Gransar H, Betancur J, Eisenberg E, Otaki Y, et al. Transient ischaemic dilation and post-stress wall motion abnormality increase risk in patients with less than moderate ischaemia: Analysis of the REFINE SPECT Registry. Eur Heart J Cardiovasc Imaging 2020;21:567-75.

16. Klein E, Miller RJH, Sharir T, Einstein AJ, Fish M, Ruddy T, et al. Automated quantitative analysis of CZT SPECT stratifies cardiovascular risk in the obese population: Analysis of the REFINE SPECT Registry. J Nucl Cardiol 2020. https://doi.org/10.1007/s1 2350-020-02334-7.

17. Buechel RR, Herzog BA, Husmann L, Burger IA, Pazhenkottil $\mathrm{AP}$, Treyer $\mathrm{V}$, et al. Ultrafast nuclear myocardial perfusion imaging on a new gamma camera with semiconductor detector technique: First clinical validation. Eur J Nucl Med Mol Imaging 2010;37:773-8.

18. Verger A, Djaballah W, Fourquet N, Rouzet F, Koehl G, Imbert L, et al. Comparison between stress myocardial perfusion SPECT recorded with cadmium-zinc-telluride and Anger cameras in various study protocols. Eur J Nucl Med Mol Imaging 2013;40:331-40.

19. Chowdhury FU, Vaidyanathan S, Bould M, Marsh J, Trickett C, Dodds K, et al. Rapid-acquisition myocardial perfusion scintigraphy (MPS) on a novel gamma camera using multipinhole collimation and miniaturized cadmium-zinc-telluride (CZT) detectors: Prognostic value and diagnostic accuracy in a realworld' nuclear cardiology service. Eur Heart J Cardiovasc Imaging 2014; 15:275-83.

20. Yokota S, Mouden M, Ottervanger JP, Engbers E, Knollema S, Timmer JR, et al. Prognostic value of normal stress-only myocardial perfusion imaging: A comparison between conventional and CZT-based SPECT. Eur J Nucl Med Mol Imaging 2016;43:296-301

21. Oldan JD, Shaw LK, Hofmann P, Phelan M, Nelson J, Pagnanelli $\mathrm{R}$, et al. Prognostic value of the cadmium-zinc-telluride camera: A comparison with a conventional (Anger) camera. J Nucl Cardiol 2016;23:1280-7.

22. Lima RSL, Peclat TR, Souza A, Nakamoto AMK, Neves FM, Souza VF, et al. Prognostic value of a faster, low-radiation myocardial perfusion SPECT protocol in a CZT camera. Int $\mathrm{J}$ Cardiovasc Imaging 2017;33:2049-56.

23. Jameria ZA, Abdallah M, Fernandez-Ulloa M, O'Donnell R, Dwivedi AK, Washburn E, et al. Analysis of stress-only imaging, comparing upright and supine CZT camera acquisition to conventional gamma camera images with and without attenuation correction, with coronary angiography as a reference. J Nucl Cardiol 2018;25:540-9.

24. Cantoni V, Green R, Acampa W, Zampella E, Assante R, Nappi C, et al. Diagnostic performance of myocardial perfusion imaging with conventional and CZT single-photon emission computed tomography in detecting coronary artery disease: A meta-analysis. J Nucl Cardiol. 2019. https://doi.org/10.1007/s12350-019-01747-3 Epub May 14.

Publisher's Note Springer Nature remains neutral with regard to jurisdictional claims in published maps and institutional affiliations. 